\title{
Erratum: Spin Hall torques generated by rare-earth thin films [Phys. Rev. B 95, 064412 (2017)]
}

Neal Reynolds $\odot$, Priyamvada Jadaun, John T. Heron, Colin L. Jermain, Jonathan Gibbons, Robyn Collette, R. A. Buhrman, D. G. Schlom, and D. C. Ralph

(Q) (Received 19 September 2019; published 7 October 2019)

DOI: 10.1103/PhysRevB.100.169901

Equation (4) in the original paper contains an incorrect factor of $\alpha$ in the second term of the numerator. The correct equation should be as follows:

$$
F_{A} \equiv\left(\frac{\gamma^{2} B\left(B+\mu_{0} M_{\mathrm{eff}}\right)^{2}-\omega^{2}\left(B+\mu_{0} M_{\mathrm{eff}}\right)}{\left(\omega^{2}-\omega_{0}^{2}\right)^{2}+\alpha^{2} \gamma^{2} \omega^{2}\left(2 B+\mu_{0} M_{\mathrm{eff}}\right)^{2}}\right) .
$$

This is a purely typographical error and does not affect any other part of the paper or the analysis.

We thank Professor A. Manchon for bringing this error to our attention. 